\title{
ABERRANT COURSE AND ANATOMICAL VARIANTS OF THE BRANCHES OF THE AORTIC ARCH
}

\author{
Hounton SED 1,2 , Laleye $\mathrm{CM}_{2,3}$, Agossou AC 1 , Adjadohoun $\mathbf{S}_{1}$, Toni $\mathrm{KWG}_{2}$, Biaou $\mathbf{O}_{1}$, Hounnou $\mathrm{GM}_{2}$. \\ 1. Medical imaging department of National University Hospital Center-Hubert Koutoukou Maga \\ (CNHU-HKM) Cotonou / BENIN. \\ 2. Laboratory of Human Anatomy of the Faculty of Health Sciences (FSS) of Cotonou / BENIN. \\ 3. Visceral surgery department of CNHU-HKM Cotonou / BENIN.
}

\begin{abstract}
The aortic arch is the segment of the thoracic aorta located between its ascending and descending portions at the level of the 4th thoracic vertebra. Its main collateral branches constitute the supra aortic trunks (SAT). We reported 4 cases of variations of these trunks. The first two cases show 2 supra aortic trunks with a right bicarotido-subclavian trunk; the third, 4 supra aortic trunks with a right vertebral-subclavian trunk and the fourth, an origin's variant and an aberrant course of the supra aortic trunks. These variants find their explanation in the embryology and are important to be known by the neurologist, the neurosurgeon, the vascular surgeon, the otolaryngologist and the radiologist for an adequate diagnosis and therapy.
\end{abstract}

Keywords: Aortic arch, SAT, Aberrant path.

\section{INTRODUCTION}

The aortic arch is the segment of the thoracic aorta located between its ascending and descending portions at the 4th thoracic vertebra level. Its collateral branches, which are called supra-aortic trunks, arise from its upper face and are from right to left, the brachiocephalic arterial trunk, the left common carotid artery and the left subclavian artery, then an inconstant branch, the median thyroid artery (Kamina, 2009). This classical origin of supra aortic trunk represents $65 \%$ to $83 \%$ in the general population (Adachi, 1928; Anson, 1963; Manyama, 2011; Acar et al., 2013; Durai et al 2014). This involves the existence of anatomical variants of the branches of the aortic arch. We report 4 cases of original variant of supra aortic trunks.

\section{Case 1}

This was a 50 -year-old female patient who was addressed to perform a chest CT scan for a pulmonary embolism suspicion. Two large supraortic trunks have been discovered, the first was a right bicarotido-subclavian trunk that branches early after a short course in brachiocephalic arterial trunk and left common carotid artery. The brachiocephalic arterial trunk branches later into right subclavian artery and right common carotid artery. The second supraortic trunk was the left subclavian artery which later gives its classic collateral branches.

\section{Case 2}

This case is similar to the first. It was a 59year-old patient who performed a chest CT

\section{RESULTS}

angiogram for the exploration of a chronic pulmonary heart.

\section{Case 3}

It was a 7-month-old male, admitted undergoing a chest angiogram for the suspicion of a cardiac malformation. Two modifications of the supra aortic trunks were discovered: 1st modification: a right vertebrasubclavian common trunk. The 2nd modification: four supra aortic trunks, including the right vertebra-subclavian common trunk, the right common carotid artery, the left common carotid arteryand the left subclavian artery. 

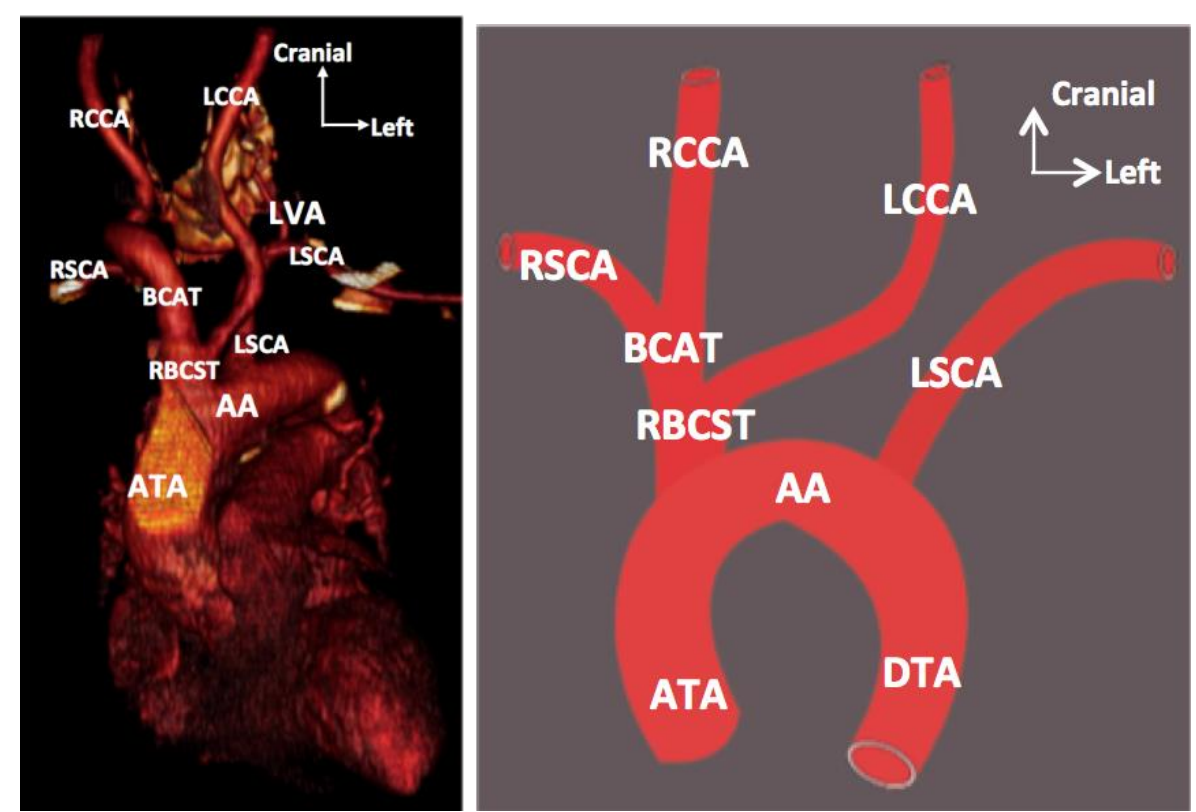

Figure 1: Ventral view of the aortic arch showing 2 supra aortic trunks including a right bicarotido-subclavian trunk (LCCA arises from the proximal part of the BCAT). ATA: Ascending thoracic aorta; AA: Aortic arch; DTA: Descending thoracic aorta; RBCST : Right bicarotido-subclavian trunk; BCAT : Brachiocephalic arterial trunk; RSCA: Right subclavian artery; RCCA: Right common carotid artery; LCCA: Left common carotid artery ; LSCA : Left subclavian artery; LVA: Left vertebral artery
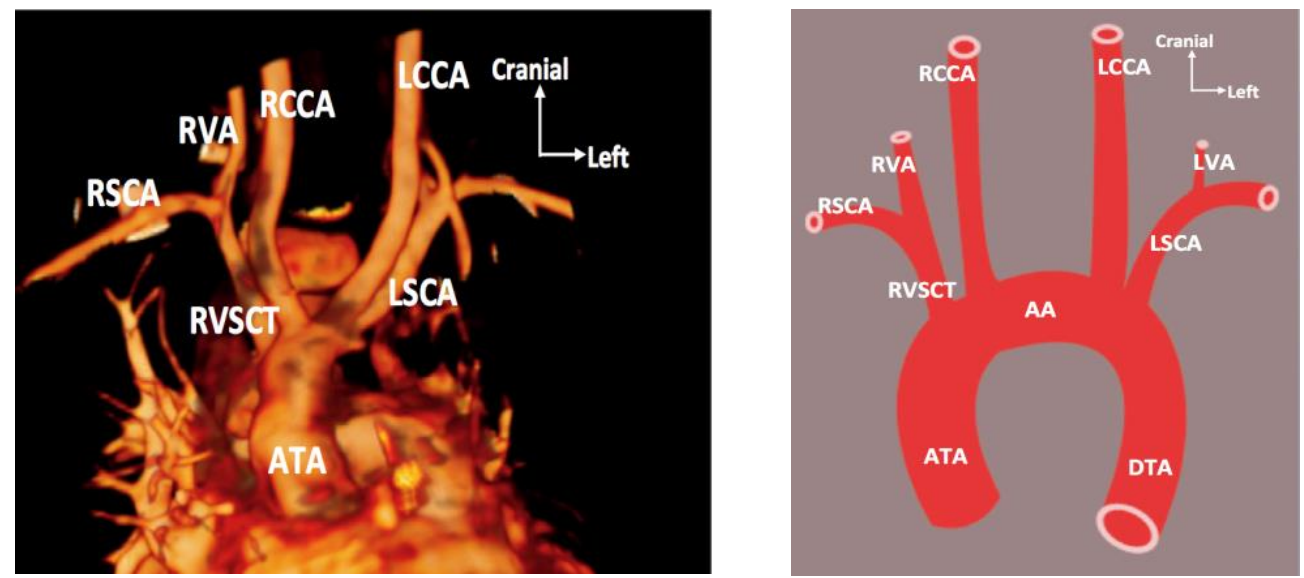

Figure 2 : Ventral view of the aortic arch showing 4 supraortic trunks including a right vertebrasubclavian trunk. ATA: Ascending thoracic aorta; AA: Aortic arch; DTA: Descending thoracic aorta; RSCA: Right subclavian artery; RCCA: Right common carotid artery; LCCA: Left common carotid artery, LSCA: Left subclavian artery; RVA: Right vertebral artery; LVA: Left vertebral artery; RVSCT: Right vertebra-subclavian trunk.

\section{Case 4}

It was a 76-year-old patient who had come to perform a chest angiogram for the suspicion of a pulmonary embolism. It was found that, on the one hand a left subclavian artery that arises between the brachiocephalic arterial trunk and the right common carotid artery. On the other hand, an aberrant course of the common carotid arteries so that the common carotid artery arising from the brachiocephalic arterial trunk on the right crosses the median line and becomes the left common carotid artery, then the one that arising directly from the aortic arch on the left, directs to the right passing behind esophagus and becomes the right common carotid. 

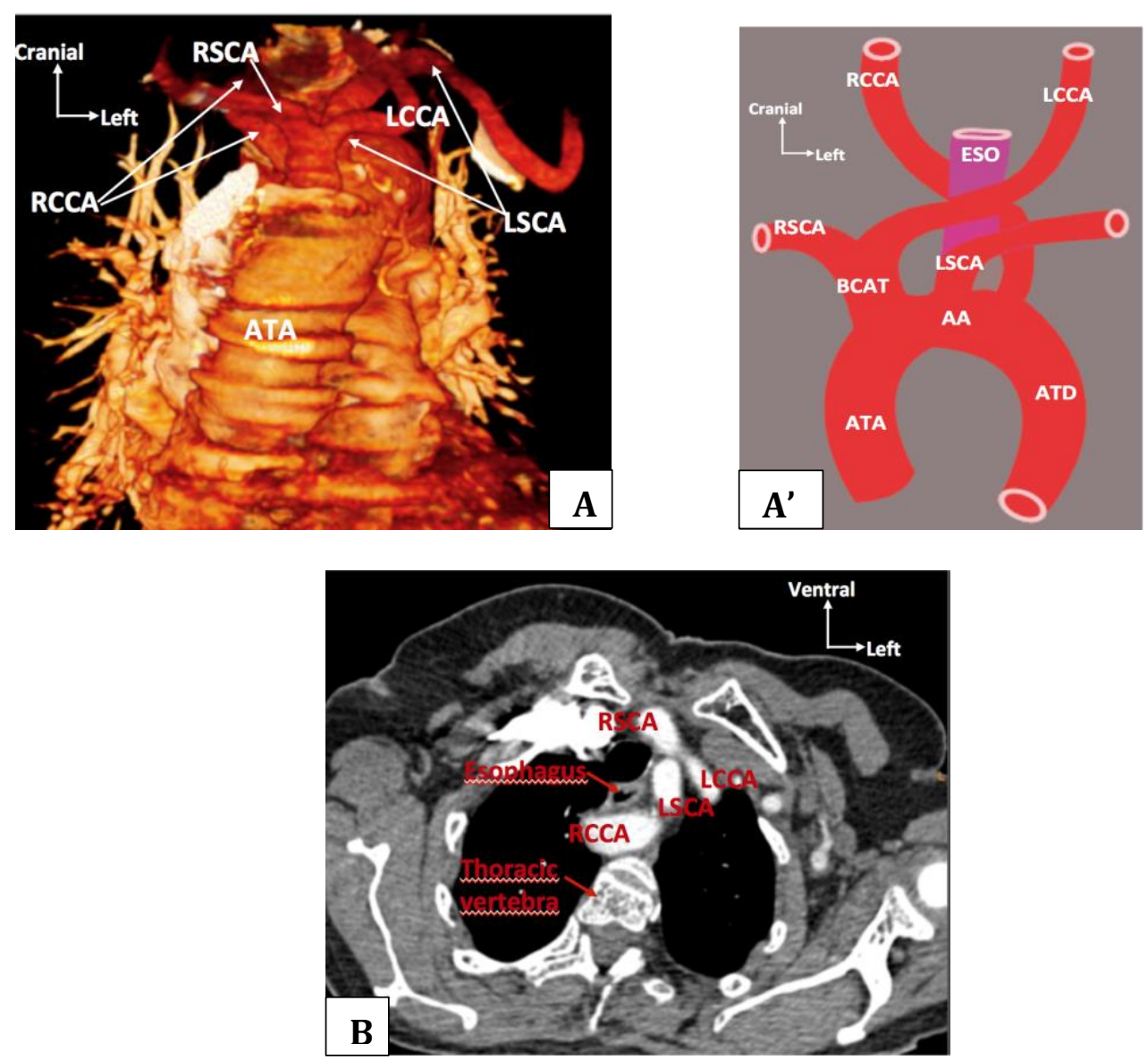

Figure 3: $A$ et $A^{\prime}$ : Ventral view showing an original variant and an aberrant path of supra aortic trunks. B: Axial section of the thorax showing the retro esophageal path of RCCA. ATA: Ascending thoracic aorta; AA: Aortic arch; DTA: Descending thoracic aorta; BCAT : Brachiocephalic arterial trunk; RSCA: Right subclavian artery; RCCA: Right common carotid artery; LCCA: Left common carotid artery, LSCA: Left subclavian artery; ESO: Esophagus

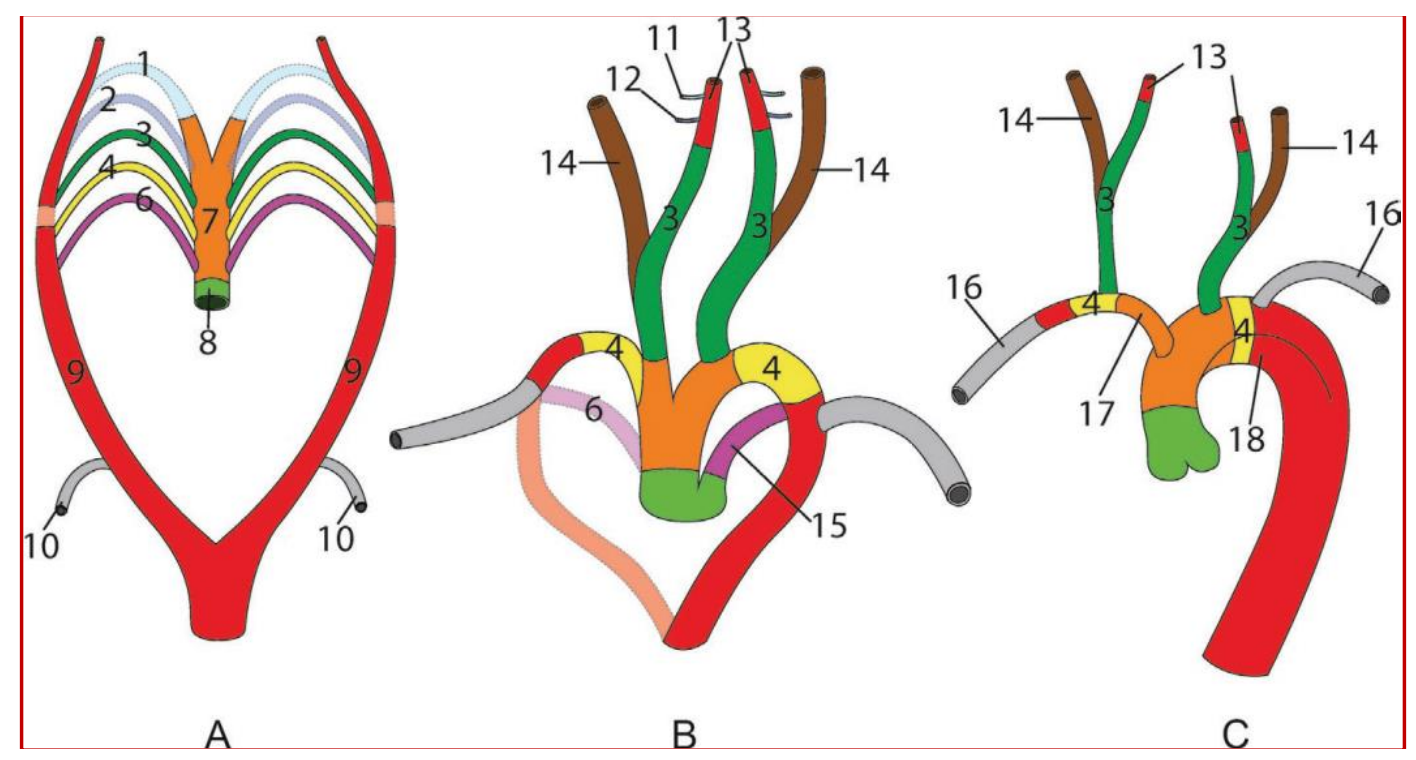

Figure 4 (A, B et C) : Development of the aortic arch [9]. 1, 2, 3, 4, 6 : aortic arches. The 5th is absent in humans. $7:$ aortic sac. $8:$ troncus arteriosus. $9:$ dorsal aorta. $10: 6$ th or 7 th segmental artery. 11 : mandibular artery derived from the 1st aortic arc. 12 : hyoidean artery derived from the 2 nd aortic arch 13 : internal carotid artery. 14 : external carotide artery. 15 : thoracic canal derived from the 6 th 


\section{DISCUSSION}

Embryology is the key to understand the different variants of the branches of the aortic arch. Indeed, during embryonic life, the aortic arches develop in the pharyngeal arches. They arise from the front of the aortic sac which continues up the truncus arteriosus, and join the dorsal aorta, right and left. The first aortic arc appears from 22nd - 24th day to involute on the 28th day, while the second appears on the 26th day to disappear 3 days later. At the 35th day, the segment of the dorsal aorta located between the 3rd and 4th aortic arches disappear, and all of the cephalic blood then passes through the only 3rd arch. The internal carotid arteries (ICA) are therefore formed by an initial segment, from the 3rd arch, and a distal segment from the dorsal aorta. External carotid arteries (ECA) develop later by budding from ICA. The involution of the 4th and 6th aortic arches occurs around the 7th week and is asymmetrical. On the left, the aortic sac remains connected to the dorsal aorta by the 4th aortic arch, these three structures giving the adult aortic arch. The 6th or 7th segmental artery, branch of the dorsal aorta, becomes the left subclavian artery (LSCA) that arise from the newly emerged aortic arch. On the right, the 6th aortic arch, and the portion of the dorsal aorta which is distal to the 6th (or 7th) segmental artery regress; the right subclavian artery (RSCA) is therefore formed from the 4th aortic arch (proximal), a short dorsal aorta segment, and the 6th (or 7th) segmental artery (distal). Finally, the portion of the arterial sac giving the 4th (future subclavian artery) and the 3rd aortic arches (future common carotid artery) is modified and becomes the brachiocephalic arterial trunk (BCAT) (Pad get, 1928; Caldemeyer et al., 1998).

The three classical supraortic trunks are found in 65 to $83 \%$ of cases. [2-6]. The common origin of BCAT and LCCA is the most common ( $25 \%$ in black, $8 \%$ in white and $13 \%$ in the general population) anatomical variation and known as the Bovine Aortic Arch configuration (Layton et al. 2006). Our first two cases highlighted a LCCA arising from the proximal part of the BCAT. This variant has a frequency of $10 \%$ in black, $5 \%$ in white and $9 \%$ in the general population [10]. It can be understood by the fact that the portion of the arterial sac giving the 4th (future subclavian artery) and 3rd right aortic arches (future common carotid artery) in its modification leading to the formation of the BCAT includes the origin of the LCCA which therefore has its definitive origin from the proximal portion of the BCAT.

The third case shows four supra aortic trunks including a right vertebra-subclavian common trunk. The vertebral artery is classically a collateral branch of the subclavian artery. A case of right vertebral-subclavian common trunk has been reported by Lemke et al (Lemke et al., 1999). This can also be formed on the left (Haifa and Wafaa, 2010). The presence of 4 supra aortic trunks has also been reported in the literature (Natsis et al., 2009). The absence of modification of the arterial sac leading to the fusion of derivatives of the 3rd (future common carotid artery) and 4th right aortic arches (future subclavian artery) which formerly were separated would explain this variant.

The 4th case showed an origin of the left subclavian artery between the BCAT and the LCCA on the one hand and an aberrant course of the common carotid arteries, especially the right which presents a retroesophageal path on the other hand. Acar et al reported a case of retro-esophageal pathway of the right common carotid in 2013 (Acar et al., 2013). The clinical interest of this aberrant path is mainly related to the possibility of dysphagia or dyspnea by compression of the esophageal and tracheal axes. The arising of the left subclavian between BCAT and LCCA could be explained by the total regression of the 4th left aortic arches leading to the direct communication of the 7th segmental artery and the left dorsal aorta with the arterial sac that gives birth to the BCAT and the LCCA. These different variants reported have essential clinical interests, especially for the vascular surgeon, the otolaryngology and the radiologist. 
In conclusion, the classical supra-aortic trunks are the brachiocephalic arterial trunk, the left common carotid artery, the left subclavian artery; all arising directly from the upper face of the aortic arch. We have reported cases of variations in origin and course of classical supra aortic trunks which find their explanation in the embryology and have to be known by the vascular surgeon, the otolaryngology and the radiologist doctor for an adequate diagnosis and therapy.

\section{REFERENCES}

1- Kamina P. 2009. Aorte In Anatomie clinique Thorax et Abdomen Tome 3. Paris, (3e édition) Maloine. 132-3.

2- Durai Pandian K, Radha K, Sundaravadhanam KVK. 2014. Study on branching pattern of arch of aorta in south Indian population. Int $\mathrm{j}$ anat res. 2:673-76.

3- Anson BH. 1963. The aortic arch and its branches. In Cardiology. Volume 1.New York: McGrawHill. 68.

4- Acar M, Ulusoy M, Zararsiz I, Efe D. 2013. Anatomical variations in the branching of human aortic arch. Biomed Res- India. 24;531-5.

5- Manyama A. 2011. A variant branching pattern of the Aortic Arch:A case report. Journal of Cardiothoracic Surgery. 6:29.

6- Adachi B. 1928. Das arterien system der Japaner, 1st ed, vol 1, Verlag der KaiserlichJapanischen Universität, Kenyusha Press, Kyoto. 29-41.

7- Padget D. 1948. The development of the cranial arteries in human embryo. Contrib Embryol. 32:207-61.

8- Caldemeyer KS, Carrico JB, Mathews VP. 1998. The radiology and embryology of anomalous arteries of the head and neck. AJR Am J Roentgenol. 170:197-203.

9- Cottier J-P, Destrieux C, Dejobert M, Geffray A, Herbreteau D. 2015. Imagerie des vaisseaux cervico-encéphaliques : radio-anatomie et variantes In Traité d'imagerie vasculaire. Paris, Elsevier Masson. 133.

10- Layton KF, Kallmes DF, Cloft HJ, Lindell EP, Cox VS. 2006. Bovine aortic arch variant in humans: Clarification of a common misnomer. AJNR Am J Neuroradiol. 27(7):1541-2.

11-Lemke AJ, Benndorf G, Liebig T, Felix R. 1999. Anomalous origin of the right vertebral artery: review of the literature and case report of the right vertebral artery origin distal to the left subclavian artery. AJNR Am J Neuroradiol. 20: 1318-21.

12- Haifa AA, Wafaa SR. 2010. An Anatomical Study of the Aortic Arch Variations. JKAU: Med. Sci. 17: 37-54.

13- Natsis KI, Tsitouridis IA, Didagelos MV, Fillipidis AA, Vlasis KG, Tsikaras PD. 2009. Anatomical variations in the branches of the human aortic arch in 633 angiographies: clinical significance and literature review. Surg Radiol Anat. 31:319-23. 